\title{
THE APHASIC PATIENT
}

\author{
by THELMA STOHR, M.A. (Log.), (Rand.).
}

Head of the Speech Therapy Department, Johannesburg Hospital.

\begin{abstract}
TOLLOWING damage to certain areas of the brain, some individuals may develop aphasia. This is the impairment or loss of the ability to use or comprehend language after it has been acquired.
\end{abstract}

Types of Aphasias:

There are two main types of this problem. (1) The sensory or receptive type, which is characterized by an inability to understand the written or spoken word.

(2) The motor or expressive type which results in the loss of power to speak write or draw. It is rare indeed to find of powividual suffering from pure motor or sensory aphasia. Usually there is a combination of the two, with a greater predominance of the motor disability.

Generally speaking, aphasia is caused by two main types of lesion. (1) Those resulting from external causes. these are usually of traumatic origin such as gunshot wounds and accidents. This type usually responds well to speech therapy especially when treatment is begun as soon as possible. (2) Aphasias of vascular origin are the commonest type found in the Speech Therapy Department. Apart from the severely deteriorated speech condition, most of these cases show a great loss of learned material. An educational estimate usually reveals a loss of even the rudiments of reading, writing and arithmetic. These cases also tend to show an intellectual deterioration as well as an inability to maintain adequate emotional control.

\section{Incidence of Aphasia:}

While traumatic' aphasics are comparatively rare in a peace time speech clinic, the incidence of vascular aphasia is quite high. There also appears to be a greater number of males as compared with females. Our files reveal that approximately $66 \%$ of patients referred for speech therapy are males. With regard to the rate of recovery however, there does not appear to be any appreciable sex difference.

In a recent survey among non-European patients it was found that the greatest number of cases referred for speech therapy were aphasics of vascular and traumatic origin. The incidence of traumatic aphasias was higher than that in the European group. It is not possible to compare the rate of recovery between the two racial groups as yet.

\section{Cerebral Dominance:}

The importance of the left cerebral hemisphere for speech and allied functions in right handed persons is an acredited fact, what ever may be its correct explanation. In the same way it has commonly been assumed that in the case of left handed persons the same importance is attached to the right hemisphere. Thus unequivocal handedness whether right or left has been thought to imply a corresponding dominance of the hemisphere contralateral to the preferred hand.

Evidence concerning aphasia and left sided hemiplegia in left handed individuals is available in the literature. However it is interesting to note that few if any lefthanded hemiplegics suffering from aphasia, have been found in the Speech Therapy Department. Most of the cases referred for speech re-education have invariably shown a greater or lesser degree of right sided hemiplegia.

In retraining, the speech therapist usually encourages the use of the unaffected hand, if the hemiplegia shows little sign of improvement. This may or may not be in conflict with the various theories on speech therapy for aphasics. The fact remains that a comparatively few patients ever regain the full use of the preferred hand again, and it is important that the patient learns to write as soon as possible, since this ability is tied up with speech and language.

\section{Speech Therapy:}

Treatment for each patient is varied to meet the individual's needs. These cover the major categories of comprehension, speaking, reading, writing and arithmetic. Before beginning therapy, several factors have to be taken into consideration. These include the length of time which has elapsed between the onset of the problem and the beginning of therapy. The longer the period, the poorer the prognosis. The premorbid educational, cultural and intellectual level must also be considered, since the treatment should be based on the patient's background. Finally a careful assessment of the loss of the language modalities must be made. This gives a pointer to prognosis and treatment.

Whether the patient is predominantly a motor or sensory type, a most important aspect must be borne in mind, namely, regardless of the extent or localisation of the injury, new association pathways must be established. Every avenue of approach is utilized in presenting material for relearning. The visual stimulus is frequently the most important. The patient sees the object, the written word, watches its formation on the therapist's lips, and then his own attempts in the mirror. The auditory stimulus is given at the same time. Listening to the therapist saying the word or sound, and then comparing it with his own is another important step. Emphasis is also placed upon the kineasthetic pattern, and the patient is made conscious of the "feel" of the sound or word.

Some of the severely amnesic cases who cannot recall long enough to say even a short word are the most difficult of all patients. Their training must consist of constant repetition of the bare rudiments of the alphabet and simple words that can be used every day. The less difficult sounds are learned first and then combined into easy words. All preliminary drills are written in a notebook as they are required so that there is a record of what has been learned. As facility is acquired, the exercises are divided into units so that there are some words at the patient's command to meet the needs of simple situations. For example a unit may be built around parts of the body, and includes such words as "thumb", "mouth", "arm", and so on. Whenever possible they are used in short sentences.

During the therapy period the problem of perseveration must be dealt with. Re-iteration of earlier taught words tend to crop up when new words are being learnt. Fatigue also affects the therapy session. Even if allowances for brief rest periods between speech attempts are made, patients often reach a point beyond which no adequate response can be elicited. As the treatment progresses this condition tends to improve to a certain extent.

Aphasia is a serious condition, and the patient is given every opportunity for speech re-education even if prognosis is poor. It is of great importance that the individual suffering from aphasia be able to express his wishes even though the words are meagre and incorrectly used. $\mathrm{He}$ is often in a state of emotional turmoil and even a poor ability to express himself affords an outlet.

Speech therapy is not necessarily the sole solution to the problem of aphasia. Teamwork between the physictherapist the occupational therapist and the social worker often produces more beneficial and lasting results. 